\title{
Comparison of Objective and Subjective Visual Quality Following LASEK, FS- LASIK and SMILE for Different Myopic Corrections: A Prospective Study
}

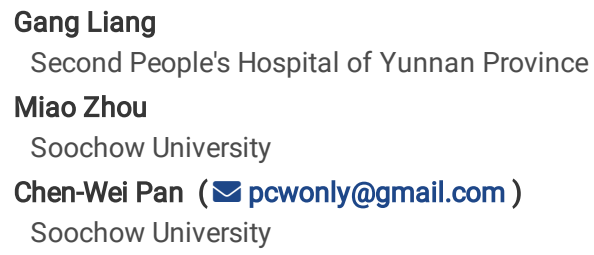

\section{Research Article}

Keywords: visual quality, LASEK, FS-LASIK, SMILE, myopia, OQAS

Posted Date: November 10th, 2021

DOI: https://doi.org/10.21203/rs.3.rs-1033639/v1

License: () (i) This work is licensed under a Creative Commons Attribution 4.0 International License. Read Full License 


\section{Abstract}

Background: To investigate the differences in visual quality after laser epithelial keratomileusis (LASEK), femtosecond laser in situ keratomileusis (FS-LASIK) and small-incision lenticule extraction (SMILE) in different degrees of myopia.

Methods: This prospective study incorporated 200 consecutive myopic subjects (200 eyes). Myopia was subdivided into 3 levels based on the preoperative spherical equivalent (SE) value: low myopia (SE $\geq-3.0 \mathrm{D}$ ), moderate myopia (-3.0 D $>\mathrm{SE} \geq-6.0 \mathrm{D}$ ), and high myopia (SE $<-6.0 \mathrm{D})$ ). Visual quality was recorded and analyzed both objectively using the Optical Quality Analysis System II (OQAS II), and subjectively with uncorrected distance visual acuity (UDVA) and a questionnaire, preoperatively, 1 month and 6 months postoperatively.

Results: Six months after LASEK, FS-LASIK and SMILE in myopia, the logarithm of the minimum angle of resolution (LogMAR) UDVA statistically decreased compared with preoperative values (all $\mathrm{P}<0.001$ ). While preoperatively, in three surgical procedure groups, objective visual quality parameters demonstrated no significant difference among three degrees of myopia (all $\mathrm{P}>0.05$ ), at 6 months postoperatively, after FS-LASIK and SMILE, the modulation transfer function (MTF) cutoff frequency in low myopia was significantly higher than that in high myopia (both $\mathrm{P}<0.05$ ). Moreover, intergroup differences in LASEK, FS-LASIK and SMILE groups were not statistically significant, before and 6 months after surgery (all P >0.05), except that FS-LASIK provided a significantly higher preoperative LogMAR UDVA than SMILE in high myopia $(P<0.05)$.

Conclusion: LASEK, FS-LASIK and SMILE could achieve similar improvement in visual quality to each other after myopia correction. Low myopic patients may achieve better postoperative visual quality after FS-LASIK and SMILE than high myopic patients.

Trial registration: This is a prospective study. The study followed the Declaration of Helsinki and was approved by the Institutional Review Board of the Second People's Hospital of Yunnan Province.

\section{Introduction}

Myopia has emerged as worldwide public health issue and uncorrected myopia is a common cause of blindness and visual impairment around the world. ${ }^{(1,2)}$ Recently, safe, predictable and effective corneal refractive surgery has become a preferred alternative for myopia correction, ${ }^{(3-5)}$ in which corneal refractive correction can mainly be accomplished by 1.surface ablation such as laser epithelial keratomileusis (LASEK) and photorefractive keratectomy (PRK), or 2.flap procedures such as femtosecond laser in situ keratomileusis (FS-LASIK) and 3.refractive lenticule extraction such as small-incision lenticule extraction (SMILE). ${ }^{(6)}$ The diversity of surgical alternatives not only provides myopic patients with a wide range of potential refractive options, but also requires surgeons to properly understand the risks-benefits for myopia.

With the development of refractive surgical technology, increasing concerns have transferred from efficacy and safety to postoperative visual quality. ${ }^{(4)}$ More recently, the Optical Quality Analysis System II (OQAS II, Visiometrics, Spain), a novel instrument based on double-pass technology, has gained popularity to assess objective visual quality. ${ }^{(7)}$ While the conventional wavefront sensors ignore the contribution of intraocular scattering on imaging and thus overestimate the visual quality, ${ }^{(8)}$ OQAS II could produce a more accurate description of the visual quality under the co-effects of aberration and scattering objectively and dynamically, ${ }^{(9)}$ presenting good repeatability and reproducibility. ${ }^{(10,11)}$ Nevertheless, there are few studies applying OQAS-II to evaluate the visual quality under corneal refractive surgery objectively. Moreover, although some studies have compared the visual quality following SMILE and FS-LASIK for myopia, ${ }^{(12-14)}$ or after SMILE and LASEK for myopia, ${ }^{(4,15)}$ few studies simultaneously compare the postoperative visual quality after LASEK, FS-LASIK and SMILE in myopia.

Accordingly, we conducted this prospective study to compare the efficacy of LASEK, FS-LASIK and SMILE on the visual quality when correcting myopia. In this study, pre- and postoperative visual quality was assessed both objectively using OQAS II, and subjectively with uncorrected distance visual acuity (UDVA) and the Quality of Life Impact of Refractive Correction (QIRC) Questionnaire. This study may serve as a primer to help surgeons and patients better weigh against the above three optical corneal refractive corrective options in terms of visual quality and may also act as a springboard for creative research topic to study specific mechanism between the selection of surgery procedures and visual quality.

\section{Patients And Methods}

\section{Subjects}

The prospective consecutive series recruited patients requiring LASEK, FS-LASIK or SMILE to correct myopia from January 2020 to December 2020 at the Department of Ophthalmology, the Second People's Hospital of Yunnan Province. Myopes were involved in this study, if they met all the following preoperative inclusion criteria: (1) aged between 18 and 40 years; (2) best corrected distance visual acuity (BCDVA) of more than 20/20; (3) sphere up to -10.00 dioptres (D) with astigmatism up to $-6.00 \mathrm{D}$; (4) minimum corneal thickness above $480 \mu \mathrm{m}$; (5) stable refraction for at least 2 years (change within $\pm 0.50 \mathrm{D}$ ); (6) no history of contact lens wear, or discontinuation of lens wear prior to surgery (soft contact lenses for at least 1 week and rigid gas permeable lenses for at least 1 month, respectively); (7) able and willing to complete QIRC questionnaire independently. Patients with systemic diseases, previous ocular surgery, history of corneal or intraocular trauma or other known ocular abnormality other than myopia were excluded. Among the initial cohort of 378 myopes received standard preoperative examination, only 230 subjects satisfied all the above inclusion criteria and finally 200 subjects were included in the analysis. These 200 patients were included randomly into three surgery groups and data from one eye of each patient were selected randomly for statistics. Myopia was subdivided into 3 levels based in these subjects on their spherical equivalent (SE) value before treatment: low myopia (SE $\geq-3.0 \mathrm{D})$, moderate myopia ( $-3.0 \mathrm{D}>\mathrm{SE} \geq-6.0 \mathrm{D})$, and high myopia ( SE <-6.0 D). ${ }^{(16)}$ The study followed the Declaration of Helsinki and was approved by the Institutional Review Board of the Second People's Hospital of Yunnan Province. Written informed consent was obtained from all participants in the study. 


\section{Surgical procedures}

All surgical procedures were performed by the same surgeon (GL). The LASEK procedures involved removing the corneal epithelium by placing the 8.0-8.5 mm trephine with $20 \%$ ethanol over the central cornea for 10-20 seconds, ablating the corneal surface by the Schwind Amaris 750 S excimer laser (Schwind EyeTech-Solutions), repositioning the epithelium, and applying a bandage contact lens (Bausch \& Lomb) for 4-6 days. As for FS-LASIK procedures, a $500 \mathrm{kHz}$ Visumax femtosecond laser system (Carl Zeiss Meditec) was applied for a hinged flap creation, and the exposed stromal bed was ablated in suit by the Schwind Amaris 750S excimer laser. Flap diameter and thickness were 7.9-8.0 mm and $110 \mu \mathrm{m}$, respectively, with standard $90^{\circ}$ hinge angle and $90^{\circ}$ angled side cut. The intentioned optic zone was set $6.5 \mathrm{~mm}$. The SMILE procedures were performed using the same femtosecond laser system as FS-LASIK with a repetition rate of $500 \mathrm{kHz}$ and pulse energy of 110-130 nJ. In SMILE procedures, a lenticule of the desired correction was created in the cornea's stroma by the femtosecond laser and then extracted through a small corneal incision, without creating a flap. The laser parameters were as followed: cap thickness, 120 m;

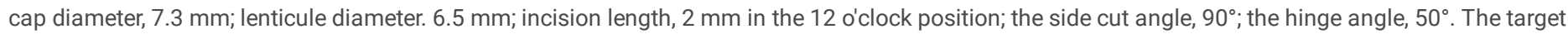
refraction of the patients was emmetropia.

For all subjects, the operated eyes were patched up transparently to the day after operation. No postoperative medication was given to the patients until the first postoperative day. Topical levofloxacin, recombinant human epidermal growth factor, $0.5 \%$ loteprednol and $0.1 \%$ sodium hyaluronate were used after all the above three surgeries.

\section{Measurements}

Prior to the surgery, dark-adapted pupil diameter (DAPD), uncorrected visual acuity (UDVA), BCDVA and SE were recorded. Moreover, subjective and objective visual quality was assessed preoperatively, and at 1 and 6 months postoperatively.

Subjective vision quality was assessed by UDVA and the QIRC questionnaire. UDVA directly affects postoperative satisfaction of patients, thus being one of the most important parameters in postoperative assessments, measured using Snellen visual acuity charts and converted to the logarithm of the minimum angle of resolution (LogMAR) for analysis. Higher LogMAR UDVA indicates poor vision. The QIRC questionnaire was developed and validated by Pesudovs et al. ${ }^{(17)}$ Different from the earlier validated scales, the refractive correction-related QIRC questionnaire adopt Rasch analysis to improve the traditional Likert scoring system by eliminating unnecessary items and transforming nonlinear raw categorical data to an properly weighted linear scale, which not only simplifies the questionnaire, but also enhances the discriminatory power of the score. ${ }^{(18)}$ The scope of the overall score is from 0 to 100 , while the higher the score, the better the QOL.

Objective visual quality was assessed by OQAS II, which performs the measurements by recording and analyzing the image from a monochromatic point source after reflection in the retina and a double pass through the ocular media. The following measurements were provided by OQAS II(19): (1) the objective scattering index (OSI): an objective index of intraocular light scattering and optical aberrations, which is defined as the ratio between the integrated light in the peripherally ring and in the central peak of the double-pass point spread function (PSF) image. Lower OSI value indicates a lower level of intraocular scattering which in turn suggests a higher visual quality. (2) the modulation transfer function (MTF) cutoff frequency: frequency in cycle per degree (cpd) at which the MTF reaches the value of 0.01 , corresponding to the lowest contrast of $1 \%$. The higher MTF cutoff frequency value denotes the better visual quality. (3) the Strehl ratio (SR): the ratio of the peak intensity from the PSF illuminance in the aberrated eye to those in the unaberrated eye. A SR value of 1.0 represents the perfect visual quality and the higher SR value, the better visual quality.

\section{Statistical analysis}

In this study, the Statistical Package for Social Sciences software package (SPSS, version 25.0 for Windows) and Graphed Prism 8.0 was used for statistically analyzing the data and compile figures, respectively. Continuous data were expressed as mean \pm standard deviation (SD) and compared between two groups with independent samples t-test as all the continuous variables were normally distributed; comparison among three groups of measurement variables was performed by one-way analysis of variance (ANOVA), and least significant difference (LSD) t-test was conducted for pairwise comparison after that. Categorical data were compared with the chi-squared test, presented as the number of patients. A difference was deemed statistically significant if $\mathrm{P}<0.05$.

\section{Results}

In this study, of 378 subjects who were eligible to participate, 230 individuals met the preoperative inclusion criteria as well as undergone the corneal refractive surgery, 222 subjects participated in the visual quality assessments at postoperative 1 month follow-up, and 206 subjects at prospective 6 months follow-up, with finally a total of 200 subjects included in the analysis (Figure 1).

Preoperative characteristics of these 200 individuals (200 eyes) are presented in Table 1. No significant difference was found in terms of age and sex among LASEK, FS-LASIK and SMILE groups $(P>0.05)$. Although the preoperative SE of moderate and high myopia was significantly different from each other in the LASEK, FS-LASIK and the SMILE group $(P<0.05)$, other comparisons of ophthalmic examination results prior to surgery among three surgical method groups exhibited no statistical significance $(P>0.05)$. 
Table 1 Baseline characteristics of study participants (Mean \pm SD).

\begin{tabular}{lllll} 
Characteristic & LASEK $(\mathrm{n}=50)$ & FS-LASIK $(\mathrm{n}=75)$ & SMILE $(\mathrm{n}=75)$ & $P$ \\
\hline Age (years) & $25.26 \pm 6.34$ & $24.31 \pm 5.45$ & $25.36 \pm 5.09$ & 0.210 \\
\hline Male/female $^{\dagger}$ & $60 / 40$ & $72 / 78$ & $78 / 72$ & 0.175 \\
\hline DAPD $(\mathrm{mm})$ & $6.39 \pm 0.64$ & $6.42 \pm 0.57$ & $6.38 \pm 0.58$ & 0.899 \\
\hline LogMAR UDVA & $1.18 \pm 0.20$ & $1.27 \pm 0.28$ & $1.23 \pm 0.19$ & 0.097 \\
\hline SE (D) & & & & \\
\hline Low Myopia & $-2.08 \pm 0.58$ & $-2.10 \pm 0.57$ & $-2.23 \pm 0.41$ & 0.569 \\
\hline Moderate Myopia & $-4.09 \pm 0.67$ & $-4.98 \pm 0.72^{\mathrm{a}}$ & $-4.60 \pm 0.78^{\mathrm{ab}}$ & 0.001 \\
\hline High Myopia $\#$ & - & $-7.32 \pm 0.88$ & $-6.85 \pm 0.53^{\mathrm{b}}$ & 0.027
\end{tabular}

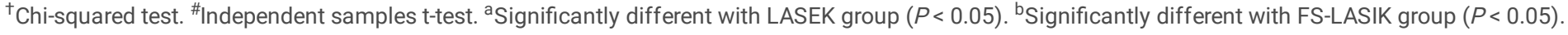

Abbreviation: DAPD = dark-adapted pupil diameter, LogMAR = the logarithm of the minimum angle of resolution, UDVA = uncorrected distance visual acuity, $\mathrm{SE}=$ spherical equivalent, $\mathrm{D}=$ diopter, LASEK = laser epithelial keratomileusis, FS-LASIK = femtosecond laser in situ keratomileusis, SMILE = small-incision lenticule extraction.

Figure 2 shows the time-dependent changes of visual quality parameters before and after surgeries. In all the three surgical procedures, the LogMAR UDVA at 6 months postoperatively statistically decreased compared with the preoperative values $(P<0.001)$. In the FS-LASIK group, the QIRC scores in low myopia and moderate myopia both significantly increased 6 months after surgery $(P<0.05)$. In terms of objective visual quality, there was no significant change in the values of OSI, SR, and MTF cutoff frequency before and after three surgical procedures $(P>0.05)$. Specifically, in high myopia, after FS-LASIK, the OSI value markedly increased from preoperatively to 1 month postoperatively and gradually declined to preoperative levels at 6 months postoperatively ( $<<0.001)$, and after FS-LASIK and SMILE, the MTF cutoff frequency noticeably showed a trend of decreasing first and then rising to preoperative levels $(P<0.05)$.

Besides, we also performed the comparisons of among different myopic levels following same surgery in Figure 2. Regarding objective visual quality indices of OQAS II, preoperatively, in three surgical procedure groups, the MTF, SR, and OSI showed no significant difference among the three degrees of myopia (P > 0.05). However, at 6 months postoperatively, in FS-LASIK group, the SR value and MTF cutoff frequency in low myopia was significantly higher than that in high myopia $(P<0.05)$; in the SMILE group, the MTF cutoff frequency in low myopia in the SMILE group was relatively higher than that in high myopia $(P<$ 0.01).

Moreover, Table 2 shows the comparisons of visual quality parameters among three different surgeries didn't exhibit significant differences before and 6 months after surgery (all $\mathrm{P}>0.05$ ), except that FS-LASIK provided a significantly higher preoperative LogMAR UDVA than SMILE in high myopia ( $<<0.05$ ). 
Table 2 Comparisons of visual quality parameters under LASEK, FS-LASIK and SMILE for myopia (Mean \pm SD).

\begin{tabular}{|c|c|c|c|c|c|c|c|c|c|c|}
\hline \multirow[t]{2}{*}{ Parameter } & \multicolumn{4}{|l|}{ Low Myopia } & \multicolumn{4}{|c|}{ Moderate Myopia } & \multicolumn{2}{|l|}{ High Myopia } \\
\hline & LASEK & FS-LASIK & SMILE & $P_{1}$ & LASEK & FS-LASIK & SMILE & $P_{1}$ & FS-LASIK & SMIL \\
\hline \multicolumn{11}{|l|}{ Preoperative } \\
\hline LogMAR UDVA & $1.12 \pm 0.26$ & $1.02 \pm 0.29$ & $1.12 \pm 0.24$ & 0.359 & $1.25 \pm 0.09$ & $1.29 \pm 0.08$ & $1.23 \pm 0.11$ & 0.060 & $1.50 \pm 0.18$ & $1.34=$ \\
\hline QIRC scores & $38.93 \pm 4.74$ & $39.08 \pm 4.37$ & $39.01 \pm 6.10$ & 0.995 & $38.24 \pm 5.43$ & $37.51 \pm 6.16$ & $38.72 \pm 5.22$ & 0.746 & $38.56 \pm 4.40$ & 38.16 \\
\hline OSI & $0.62 \pm 0.42$ & $0.55 \pm 0.39$ & $0.63 \pm 0.27$ & 0.705 & $0.72 \pm 0.46$ & $0.58 \pm 0.35$ & $0.61 \pm 0.42$ & 0.401 & $0.76 \pm 0.61$ & $0.83:$ \\
\hline SR & $0.26 \pm 0.08$ & $0.23 \pm 0.07$ & $0.25 \pm 0.08$ & 0.298 & $0.23 \pm 0.06$ & $0.23 \pm 0.07$ & $0.21 \pm 0.07$ & 0.636 & $0.21 \pm 0.06$ & $0.24:$ \\
\hline $\begin{array}{l}\text { MTF cutoff } \\
\text { frequency } \\
\text { (cpd) }\end{array}$ & $39.61 \pm 7.28$ & $41.79 \pm 7.64$ & $40.34 \pm 7.00$ & 0.564 & $41.00 \pm 8.22$ & $38.56 \pm 6.19$ & $38.35 \pm 6.85$ & 0.349 & $38.70 \pm 8.07$ & 38.72 \\
\hline \multicolumn{11}{|l|}{1 month } \\
\hline LogMAR UDVA & $0.00 \pm 0.05$ & $0.01 \pm 0.06$ & $-0.01 \pm 0.04$ & 0.515 & $0.03 \pm 0.07$ & $0.02 \pm 0.05$ & $-0.02 \pm 0.04^{\mathrm{ab}}$ & 0.003 & $0.04 \pm 0.06$ & $0.02:$ \\
\hline QIRC scores & $39.36 \pm 5.28$ & $39.87 \pm 3.78$ & $40.44 \pm 4.67$ & 0.713 & $38.39 \pm 4.58$ & $39.74 \pm 4.23$ & $40.37 \pm 5.29$ & 0.323 & $38.91 \pm 4.45$ & 38.85 \\
\hline OSI & $0.84 \pm 0.52$ & $0.57 \pm 0.40$ & $0.85 \pm 0.48$ & 0.066 & $1.18 \pm 0.78$ & $0.98 \pm 0.48$ & $0.77 \pm 0.56$ & 0.073 & $1.69 \pm 0.68$ & $0.92:$ \\
\hline SR & $0.24 \pm 0.06$ & $0.23 \pm 0.06$ & $0.23 \pm 0.06$ & 0.790 & $0.20 \pm 0.06$ & $0.20 \pm 0.05$ & $0.24 \pm 0.06^{a}$ & 0.010 & $0.20 \pm 0.05$ & $0.21=$ \\
\hline $\begin{array}{l}\text { MTF cutoff } \\
\text { frequency } \\
\text { (cpd) }\end{array}$ & $39.12 \pm 8.36$ & $37.26 \pm 8.22$ & $39.73 \pm 9.01$ & 0.570 & $36.15 \pm 12.55$ & $36.76 \pm 7.69$ & $37.14 \pm 10.36$ & 0.945 & $29.51 \pm 9.18$ & 31.31 \\
\hline
\end{tabular}

\begin{tabular}{|c|c|c|c|c|c|c|c|c|c|c|}
\hline \multicolumn{11}{|l|}{6 months } \\
\hline LogMAR UDVA & $0.03 \pm 0.06$ & $0.00 \pm 0.06$ & $-0.01 \pm 0.06$ & 0.055 & $-0.02 \pm 0.05$ & $0.02 \pm 0.06$ & $0.01 \pm 0.08$ & 0.079 & $-0.01 \pm 0.05$ & -0.02 \\
\hline QIRC scores & $41.36 \pm 2.40$ & $42.17 \pm 4.44$ & $41.69 \pm 5.03$ & 0.784 & $39.82 \pm 3.07$ & $40.85 \pm 3.33$ & $41.78 \pm 3.39$ & 0.110 & $40.29 \pm 5.27$ & 40.69 \\
\hline OSI & $0.66 \pm 0.38$ & $0.68 \pm 0.35$ & $0.64 \pm 0.36$ & 0.941 & $0.89 \pm 0.60$ & $0.83 \pm 0.57$ & $0.89 \pm 0.61$ & 0.929 & $0.78 \pm 0.53$ & $0.90=$ \\
\hline SR & $0.24 \pm 0.07$ & $0.25 \pm 0.08$ & $0.25 \pm 0.06$ & 0.892 & $0.23 \pm 0.06$ & $0.22 \pm 0.06$ & $0.23 \pm 0.06$ & 0.708 & $0.20 \pm 0.06$ & $0.23=$ \\
\hline $\begin{array}{l}\text { MTF cutoff } \\
\text { frequency } \\
\text { (cpd) }\end{array}$ & $40.83 \pm 6.37$ & $40.29 \pm 5.69$ & $40.94 \pm 6.59$ & 0.926 & $39.94 \pm 6.50$ & $38.20 \pm 5.56$ & $39.21 \pm 7.05$ & 0.629 & $36.48 \pm 6.20$ & 36.35 \\
\hline
\end{tabular}

$P_{1}$ was calculated by one-way analysis of variance with LSD corrections. $P_{2}$ was calculated by independent t test. a Significantly different with LASEK group (P

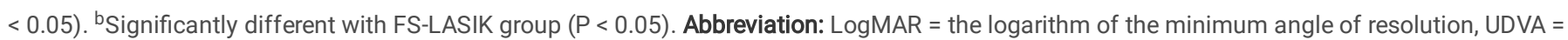
uncorrected distance visual acuity, QIRC = quality of life impact of refractive correction, OSI = objective scattering index, SR = strehl ratio, MTF = modulate transfer function, $\mathrm{cpd}$ = cycle per degree, LASEK = laser epithelial keratomileusis, FS-LASIK = femtosecond laser in situ keratomileusis, SMILE = small-incision lenticule extraction.

\section{Discussion}

In this study, we compared the subjective and objective visual quality before and after LASEK, FS-LASIK and SMILE for myopia. LASEK, FS-LASIK and SMILE all led to significant increase of subjective visual quality 6 months after surgery, but brought little impact on objective visual quality in which the difference was significant between patients with low myopia and high myopia. Besides, preoperatively and 6 months postoperatively, no statistical intergroup differences were detected in the comparisons of visual quality among three types of corneal refractive surgical techniques.

Interestingly, our results indicated that the subjective visual quality was improved after all the three surgical procedures with no statistical change detected in objective visual quality although values did vary among individuals. The improvement of subjective visual quality after three procedures has been supported by an abundant resource of evidence, ${ }^{(20-22)}$ while the UDVA may be mainly improved by laser correction with modifying the cornea shape and adjusting the focus of light which enters through the central cornea, ${ }^{(20)}$ together with the retinal or neural factors, ${ }^{(21)}$ indirectly increasing the QIRC scores. ${ }^{(23)}$ Although in our study, there was no significant change in objective visual quality parameters before and after LASEK, FS-LASIK and SMILE, it is worth noting that in all three procedures, objective visual quality decreased first and then showed an upward trend over time, with significant or inconspicuous differences, in agreement with the previous findings. ${ }^{(24-26)}$ Considering that aberration and intraocular scattering are critical factors in determining the objective optical quality after refractive surgery, $(27,28)$ the immediate decline in objective visual quality may be the result of the increment of the ocular aberrations and forward intraocular scattering, $(4,27,28)$ attributed to the following major factors: 1 .surgical reasons: intraoperative flattening the cornea and damaging the tear film stability, ${ }^{(4)}$ as well as 2.incision healing response: postoperative remodeling corneal shape ${ }^{(29)}$ and rising the transient corneal haze. ${ }^{(30)}$ Nevertheless, the limited 6 months follow-up and the trend of rising in objective visual quality at the end of the follow-up suggest that the conclusion is far from conclusive and the visual quality may be expected to continue increasing beyond 6 months. Further studies comparing the long-term visual quality are warranted. 
We also found that despite not significantly different before surgery, the objective visual quality parameters, especially the MTF cutoff frequency, decreased as the degree of myopia increased after FS-LASIK and SMILE significantly. Similarly, Paquin et al indicated a quasi-linear correlation between aberration and SE.

${ }^{(31)}$ Generally, the MTF cutoff frequency could reflect all the optical characteristics of the human eye, including the overall optical defects involved in retinal image degeneration, such as aberrations, scattering, and diffraction. ${ }^{(32)}$ Hence, the explanation may be that compared with low myopes, high myopes might entail deeper ablation subsequently inducing greater inflammation under FS-LASIK, ${ }^{(33)}$ and more Bowman's membrane microdisortions under SMILE, ${ }^{(34)}$ together with longer tissue remodeling and wound healing processes. ${ }^{(35)}$ Besides, previous studies have reported that there are less predictable outcomes and more risk of corneal ectasia in high myopic patients after laser refractive surgery. ${ }^{(36)}$ Therefore, patients with high myopia may not be proper candidates for corneal refractive surgery. However, because of the greater functional impairment undergone by high myopes, the potential limitations of corneal refractive surgery might be still more acceptable. ${ }^{(37)}$ On the other hand, substantial novel surgical techniques for high myopia correction, such as the implantation of intraocular lens, have advanced over the past few years, ${ }^{(38)}$ and may provide greater reversibility or adjustability than corneal refractive procedures. ${ }^{(6)}$ With these emerging trends, it is necessary for ophthalmologists to understand the pros and cons of each refractive surgery and weigh against optical corrective surgical options, with careful and appropriate patient selection. ${ }^{(37)}$

Despite that a couple of studies have been done on pairwise comparisons of visual quality between every two surgical procedures among LASEK, FS-LASIK and SMILE, $(4,13,15)$ anecdotally, this study is the first to make comprehensive visual quality comparisons among all these three surgery techniques for different degrees of myopia, both objectively and subjectively. In our analysis, no significant intergroup differences were found in the comparisons of visual quality among LASEK, FS-LASIK and SMILE preoperatively and 6 months postoperatively, except in preoperative UDVA of high myopia, which has been supported by pairwise comparison results of previous studies. ${ }^{(6,15)}$ Therefore, LASEK, FS-LASIK and SMILE may be equally effective in visual quality improvement, and the selection of surgical procedures could rely on individual patient characteristics or surgeon preferences.

A major strength of our study is that we used OQAS system to estimate objective visual quality with good repeatability and reproducibility. ${ }^{(7)}$ Although Hartmann-Shack wavefront sensors has been the most commonly used tool for visual outcome assessments, it is well known that the wavefront aberrometers overestimate visual quality in eyes where high-order aberrations and scatter are obvious due to limitation imposed by lens sampling. ${ }^{(8)}$ Thus, in our study, for the objective visual quality estimation in eyes after corneal refractive surgeries where different levels of scattering exists because of differences in the degree of corneal haze, ${ }^{(39)}$ the double-pass OQAS II may be a more appropriate option due to the property of the double-pass approach of capturing the comprehensive information on the retinal image which renders the technique extremely powerful in many of the conditions that specially influence scattering.

However, the findings of our study must be interpreted in the context of the following important limitations. First, the preoperative SE was statistically different between the SMILE and FS-LASIK groups for high myopic correction, as well as between three surgical procedures for moderate myopic correction, which might suggest a probable imbalance among the three surgical procedures because of different ablation depth, and thus affect outcomes. Secondly, this study did not collect sufficient long-term follow-up data. Although in our study, there was no significant change in objective visual quality preoperatively and 6 months postoperatively, there was a trend of rising at 6 months after surgery. Thus, our study may not be representative of the eventual postoperative visual quality level after surgery. Thirdly, there is absence of data in high myopia under LASEK, mainly due to the fact that clinically, high myopic patients generally choose intraocular surgery to correct myopia compared with LASEK, so it is difficult to collect data in high myopia under LASEK.

\section{Conclusions}

In conclusion, visual quality was similarly accelerated with LASEK, FS-LASIK and SMILE, with no significant differences between the three surgeries. Besides, the improvement effect on visual quality is more prominent for low and moderate myopia than for high myopia. These findings may provide a valuable guidance for ophthalmologists to properly understand the risks-benefits of each corneal refractive surgery for myopia and appropriately select candidates to subsequently yield better visual quality. Further large sample and high-quality randomized controlled trials with an adequate length of follow-up are warranted.

\section{Abbreviations}

LASEK = laser epithelial keratomileusis, FS-LASIK = femtosecond laser in situ keratomileusis, SMILE = small-incision lenticule extraction, LogMAR = the logarithm of the minimum angle of resolution, UDVA = uncorrected distance visual acuity, BCDVA = best corrected distance visual acuity, QIRC = quality of life impact of refractive correction, $\mathrm{OSI}=$ objective scattering index, $\mathrm{SR}=$ strehl ratio, $\mathrm{MTF}=$ modulate transfer function, $\mathrm{cpd}=\mathrm{cycle}$ per degree, $\mathrm{DAPD}=\mathrm{dark}-$ adapted pupil diameter, SE = spherical equivalent, D = diopter, OQAS II = the Optical Quality Analysis System II.

\section{Declarations}

Ethics approval and consent to participate: The study was approved by the Institutional Review Board of the Second People's Hospital of Yunnan Province. Written informed consent was obtained from all participants in the study. All methods were performed in accordance with the relevant guidelines and regulations.

Consent for publication: Not applicable. 
Availability of data and materials: The study followed the Declaration of Helsinki and was approved by the Institutional Review Board of the Second People's Hospital of Yunnan Province. All subjects had signed the informed consents.

Competing interests: The authors declare that they have no competing interests.

Funding: the Training Program for Medical Leaders of Yunnan Health Commission [Grant Number: D-2018016]

Authors' contributions: GL and CWP contributed substantially to the conception and design of the study. GL conducted the examinations and data collection. MZ drafted the manuscript and carried out the statistical analysis. All authors read and approved the final manuscript.

Acknowledgements: Not applicable.

\section{References}

1. Bourne RR, Stevens GA, White RA, Smith JL, Flaxman SR, Price H, et al. Causes of vision loss worldwide, 1990-2010: a systematic analysis. The Lancet Global health 2013; 1:e339-49.

2. Holden BA, Fricke TR, Wilson DA, Jong M, Naidoo KS, Sankaridurg P, et al. Global Prevalence of Myopia and High Myopia and Temporal Trends from 2000 through 2050. Ophthalmology 2016; 123:1036-42.

3. Sakimoto T, Rosenblatt MI, Azar DT. Laser eye surgery for refractive errors. Lancet (London, England) 2006; 367:1432-47.

4. Zhu X, Zou L, Yu M, Qiu C, Chen M, Dai J. Comparison of postoperative visual quality after SMILE and LASEK for high myopia: A 1-year outcome. PloS one 2017 ; 12 :e0182251.

5. Morgan IG, Ohno-Matsui K, Saw SM. Myopia. Lancet (London, England) 2012; 379:1739-48.

6. Chuck RS, Jacobs DS, Lee JK, Afshari NA, Vitale S, Shen TT, et al. Refractive Errors \& Refractive Surgery Preferred Practice Pattern(R). Ophthalmology 2018; 125:P1-P104.

7. Artal P, Marcos S, Navarro R, Williams DR. Odd aberrations and double-pass measurements of retinal image quality. Journal of the Optical Society of America A, Optics, image science, and vision 1995; 12:195-201.

8. Herbaut A, Liang H, Rabut G, Trinh L, Kessal K, Baudouin C, et al. Impact of Dry Eye Disease on Vision Quality: An Optical Quality Analysis System Study. Translational vision science \& technology 2018; 7:5.

9. Chu MF, Hui N, Wang CY, Yu L, Ma B, Li Y, et al. Early outcomes of vision and objective visual quality analysis after cataract surgery with trifocal intraocular lens implantation. International journal of ophthalmology 2019; 12:1575-81.

10. Tian M, Miao H, Shen Y, Gao J, Mo X, Zhou X. Intra- and Intersession Repeatability of an Optical Quality and Intraocular Scattering Measurement System in Children. PloS one 2015; 10:e0142189.

11. Xu CC, Xue T, Wang QM, Zhou YN, Huang JH, Yu AY. Repeatability and reproducibility of a double-pass optical quality analysis device. PloS one 2015; 10:e0117587.

12. Han T, Xu Y, Han X, Zeng L, Shang J, Chen X, et al. Three-year outcomes of small incision lenticule extraction (SMILE) and femtosecond laser-assisted laser in situ keratomileusis (FS-LASIK) for myopia and myopic astigmatism. The British journal of ophthalmology 2019; 103:565-8.

13. Liu T, Lu G, Chen K, Kan Q, Bai J. Visual and optical quality outcomes of SMILE and FS-LASIK for myopia in the very early phase after surgery. BMC ophthalmology 2019; 19:88.

14. Chen X, Wang Y, Zhang J, Yang SN, Li X, Zhang L. Comparison of ocular higher-order aberrations after SMILE and Wavefront-guided Femtosecond LASIK for myopia. BMC ophthalmology 2017; 17:42.

15. Yu M, Chen M, Wang B, Zou L, Zhu X, Dai J. Comparison of Visual Quality After SMILE and LASEK for Mild to Moderate Myopia. Journal of refractive surgery (Thorofare, NJ: 1995) 2015; 31:795-800.

16. Kim MJ, Lee EJ, Kim TW. Peripapillary retinal nerve fibre layer thickness profile in subjects with myopia measured using the Stratus optical coherence tomography. The British journal of ophthalmology 2010; 94:115-20.

17. Pesudovs K, Garamendi E, Elliott DB. The Quality of Life Impact of Refractive Correction (QIRC) Questionnaire: development and validation. Optometry and vision science: official publication of the American Academy of Optometry 2004; 81:769-77.

18. leong A, Hau SC, Rubin GS, Allan BD. Quality of life in high myopia before and after implantable Collamer lens implantation. Ophthalmology 2010; 117:2295-300.

19. Saad A, Saab M, Gatinel D. Repeatability of measurements with a double-pass system. Journal of cataract and refractive surgery 2010; $36: 28-33$.

20. Sandoval HP, Donnenfeld ED, Kohnen T, Lindstrom RL, Potvin R, Tremblay DM, et al. Modern laser in situ keratomileusis outcomes. Journal of cataract and refractive surgery $2016 ; 42: 1224-34$.

21. Vilaseca M, Padilla A, Ondategui JC, Arjona M, Güell JL, Pujol J. Effect of laser in situ keratomileusis on vision analyzed using preoperative optical quality. Journal of cataract and refractive surgery 2010; 36:1945-53.

22. Kang MJ, Hwang J, Chung SH. Comparison of Corneal Wavefront-optimized and Wavefront-guided Alcohol-assisted Photorefractive Keratectomy Using Schwind Amaris 750S Laser for Myopia. Korean journal of ophthalmology: KJO 2020; 34:210-8.

23. Maraghechi G, Ojaghi H, Amani F, Moghadam TZ. Pentacam Indices in Photorefractive Keratectomy Surgery. Journal of medicine and life 2020; 13:5239. 
24. Lorente-Velazquez A, Nieto-Bona A, Collar CV, Gutierrez Ortega AR. Intraocular straylight and contrast sensitivity (1/2) and 6 months after laser in situ keratomileusis. Eye \& contact lens 2010; 36:152-5.

25. Miao H, Tian M, Xu Y, Chen Y, Zhou X. Visual Outcomes and Optical Quality After Femtosecond Laser Small Incision Lenticule Extraction: An 18-Month Prospective Study. Journal of refractive surgery 2015; 31:726-31.

26. Beerthuizen JJ, Franssen L, Landesz M, van den Berg TJ. Straylight values 1 month after laser in situ keratomileusis and photorefractive keratectomy. Journal of cataract and refractive surgery 2007; 33:779-83.

27. Oliveira CM, Ferreira A, Franco S. Wavefront analysis and Zernike polynomial decomposition for evaluation of corneal optical quality. Journal of cataract and refractive surgery $2012 ; 38: 343-56$.

28. McAlinden C, McCartney M, Moore J. Mathematics of Zernike polynomials: a review. Clinical \& experimental ophthalmology 2011;39:820-7.

29. Kwon Y, Bott S. Postsurgery corneal asphericity and spherical aberration due to ablation efficiency reduction and corneal remodelling in refractive surgeries. Eye (London, England) 2009; 23:1845-50.

30. van de Pol C, Soya K, Hwang DG. Objective assessment of transient corneal haze and its relation to visual performance after photorefractive keratectomy. American journal of ophthalmology 2001; 132:204-10.

31. Paquin MP, Hamam H, Simonet P. Objective measurement of optical aberrations in myopic eyes. Optometry and vision science: official publication of the American Academy of Optometry 2002; 79:285-91.

32. Diaz-Douton F, Benito A, Pujol J, Arjona M, Guell JL, Artal P. Comparison of the retinal image quality with a Hartmann-Shack wavefront sensor and a double-pass instrument. Investigative ophthalmology \& visual science 2006; 47:1710-6.

33. Sachdev GS. Commentary: Comparison of changes in refractive error and corneal curvature following small-incision lenticule extraction and femtosecond laser-assisted in situ keratomileusis surgery. Indian journal of ophthalmology 2018; 66:1567-8.

34. Yao P, Zhao J, Li M, Shen Y, Dong Z, Zhou X. Microdistortions in Bowman's layer following femtosecond laser small incision lenticule extraction observed by Fourier-Domain OCT. Journal of refractive surgery (Thorofare, NJ: 1995) 2013; 29:668-74.

35. Serrao S, Lombardo G, Ducoli P, Lombardo M. Optical performance of the cornea six years following photorefractive keratectomy for myopia. Investigative ophthalmology \& visual science $2011 ; 52: 846-57$.

36. Randleman JB, Woodward M, Lynn MJ, Stulting RD. Risk assessment for ectasia after corneal refractive surgery. Ophthalmology 2008; 115:37-50.

37. Kim TI, Alió Del Barrio JL, Wilkins M, Cochener B, Ang M. Refractive surgery. Lancet (London, England) 2019; 393:2085-98.

38. Alfonso JF, Fernandez-Vega L, Fernandes P, Gonzalez-Meijome JM, Montes-Mico R. Collagen copolymer toric posterior chamber phakic intraocular lens for myopic astigmatism: one-year follow-up. Journal of cataract and refractive surgery 2010; 36:568-76.

39. Zhao LQ, Zhu H, Li LM. Laser-Assisted Subepithelial Keratectomy versus Laser In Situ Keratomileusis in Myopia: A Systematic Review and Meta-Analysis. ISRN ophthalmology 2014; 2014:672146.

\section{Figures}




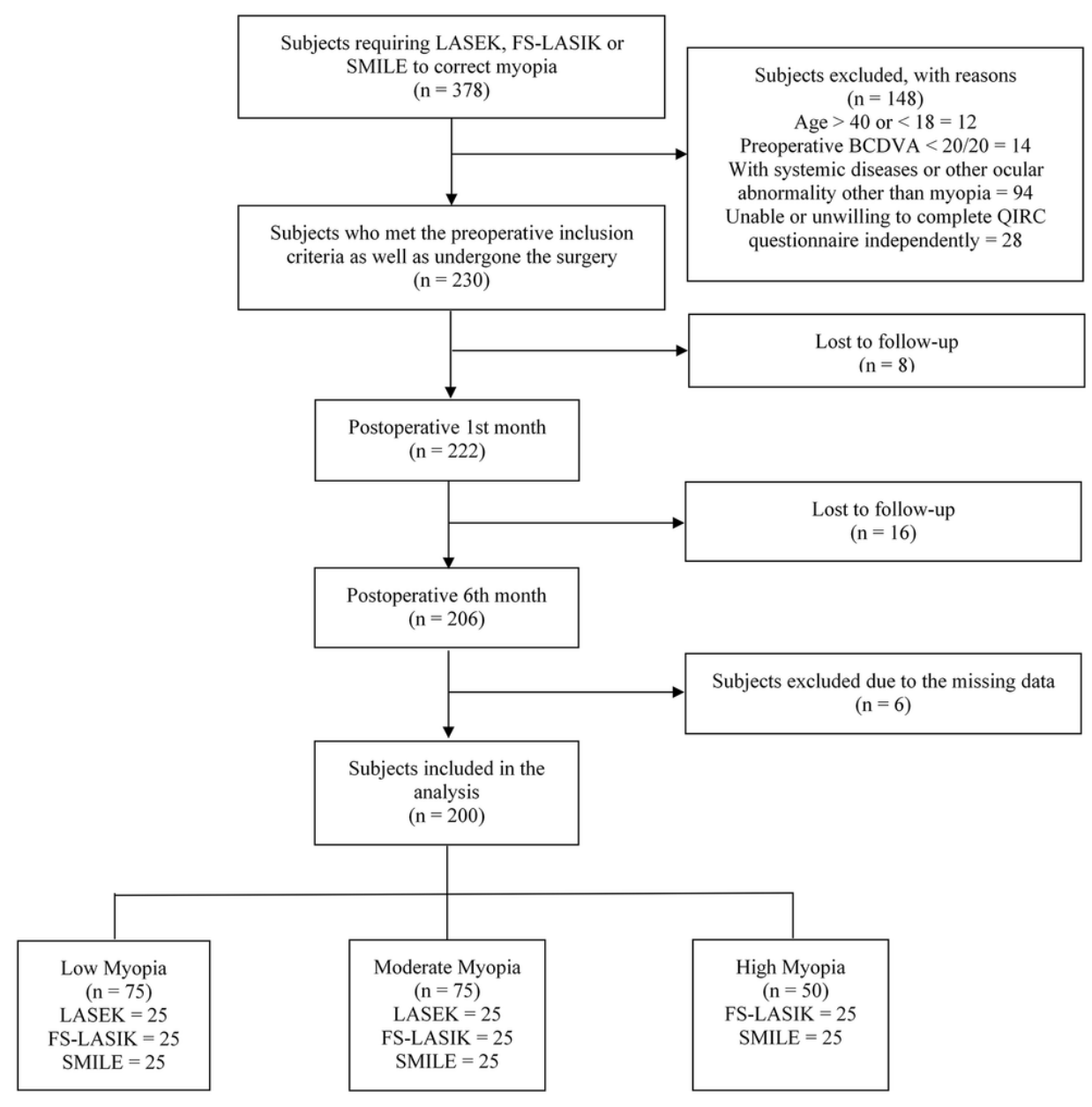

\section{Figure 1}

Flowchart for the study design $(n=200)$. Abbreviation: BCDVA = best corrected distance visual acuity, LASEK = laser epithelial keratomileusis, FS-LASIK = femtosecond laser in situ keratomileusis, SMILE = small-incision lenticule extraction . 

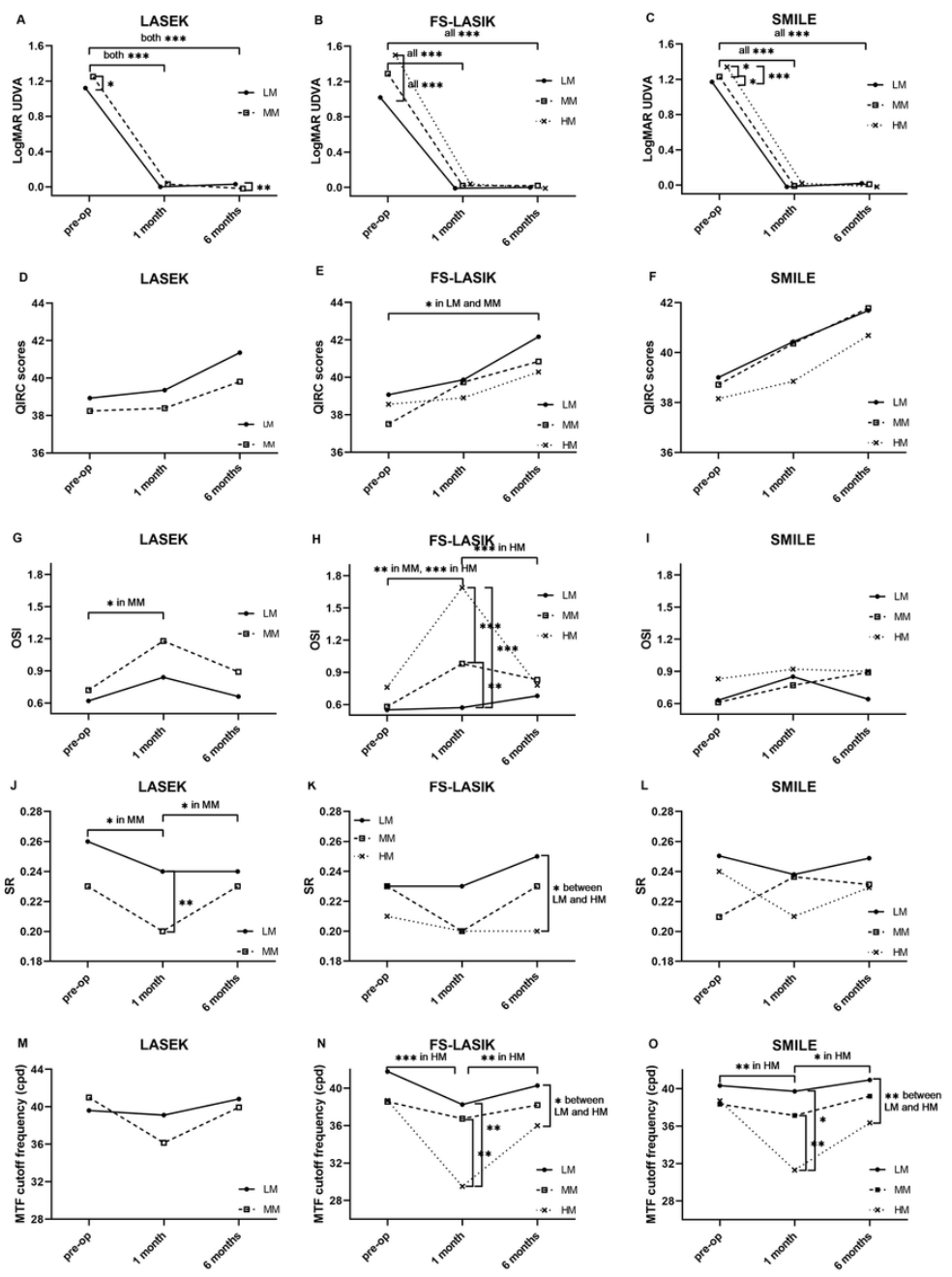

\section{Figure 2}

Time-dependent changes of visual quality parameters before and after surgery. Legend: LM Group, MM Group, HM Group. Abbreviation: *P $<0.05$, ** $\mathrm{P}<0.1$, ***P $<0.001$. LM = low myopia, MM = moderate myopia, $\mathrm{HM}=$ high myopia, LogMAR = the logarithm of the minimum angle of resolution, UDVA = uncorrected distance visual acuity, QIRC = quality of life impact of refractive correction, OSI = objective scattering index, MTF = modulate transfer function, cpd = cycle per degree, SR = strehl ratio, LASEK = laser epithelial keratomileusis, FS-LASIK = femtosecond laser in situ keratomileusis, SMILE = small-incision lenticule extraction. 